\title{
Application of immunoblotting to detect soluble Pneumocystis carinii antigen(s) in bronchoalveolar lavage of patients with Pneumocystis pneumonia and AIDS
}

\author{
K K Sethi
}

\begin{abstract}
The technique of immunoblotting for detecting soluble Pneumocystis carinii antigen(s) in bronchoalveolar lavage fluid specimens from patients with AIDS and Pneumocystis pneumonia was evaluated. A soluble 67 kilodalton polypeptide that was immunoreactive with an anti-P carinii monoclonal antibody (2G2) was found in the supernatants of 26 lavage samples from patients with pneumocystosis. Intact organisms in lavage sediments were detected by methenamine silver or immunofluorescence staining procedures. The diagnostic use of this technique was shown in four cases in which lavage sediments proved negative for intact Pneumocystis carinii organisms on first examination; 2 G2 2 reactive soluble antigen, however, was identified in the immunoblots of the supernatants from the same samples.

It is concluded that immunoblotting of bronchoalveolar lavage specimens using 2G2 monoclonal antibody as a detection probe may be a useful adjunct to the morphological demonstration of organisms by special staining procedures.
\end{abstract}

Pneumocystis carinii is responsible for life threatening pneumonia in the immune deficient $t^{12}$ and has emerged as the leading cause of morbidity and mortality in patients with AIDS. ${ }^{13}$ This organism has not been grown in cell free media and limited success has been achieved in maintaining or growing $P$ carinii of human or rat origin in certain types of cell cultures ${ }^{4-6}$; to date, continuous culturing of the organism has been not documented.

The clinical manifestations of $P$ carinii pneumonia in man are non-specific and can mimic diseases of other aetiologies. ${ }^{1}$ Definitive laboratory diagnosis of $P$ carinii infection currently relies mainly on the microscopic demonstration of a characteristic "stage"namely, cysts in infected lung tissues or respiratory fluids. ${ }^{78} P$ carinii cysts can be identified either with the aid of conventional histological stains, especially methenamine silver stain, ${ }^{8}$ or by immunofluorescence using specific monoclonal antibodies. ${ }^{9}$ Regardless of the staining methods the morphological visual- isation of these cysts is generally difficult in specimens which are not properly collected or in instances where only a few organisms are present. A test designed to detect the presence of specific $P$ carinii antigen(s) in body fluids might therefore facilitate diagnosis. There have been reports of the presence of circulating $P$ carinii antigens being shown in the sera of patients by counterimmunoelectrophoresis, ${ }^{1011}$ enzyme linked immunosorbent assay, ${ }^{12}$ and by latex agglutination, ${ }^{11}$ but their clinical utility is controversial. $^{713}$

\section{Methods}

Bronchoalveolar lavage (BAL) samples from 26 patients with AIDS and both clinical and microscopic evidence of $P$ carinii pneumonia were used. All these patients showed characteristic respiratory symptoms such as dyspnoea, non-productive cough etc, and had abnormal chest radiographs (interstitial or alveolar shadows) and reduced diffusing capacity for carbon monoxide. BAL specimens from 10 patients with bronchial asthma but no clinical or microbiological evidence of $P$ carinii infection served as matched controls. BAL fluid $(20 \mathrm{ml})$ from each patient was available for testing. After removing mucus and other debris the BAL specimens were centrifuged (10 000 $\times g$ for 20 minutes), the sediments were used for preparing microscopic slide smears, and the decanted supernatants used for immunoblotting.

A hybridoma cell line (2G2) producing monoclonal antibodies directed specifically against human $P$ carinii was obtained from $\mathrm{Dr}$ Kovacs. The hybridoma cells were injected in $\mathrm{BALB} / \mathrm{c}$ mice treated with pristane and ascitic fluids were collected. The antibody purified from ascitic fluids was of IgG $2 b$ isotype. A high titred polyclonal antiserum to human $P$ carinii was prepared in rabbits by three repeated subcutaneous injections with purified organisms emulsified in incomplete Freund's adjuvant, followed by a booster injection of antigen without adjuvant after six weeks. The IgG portion of the immune serum was purified by affinity chromatography on a staphylococcal protein A sepharose CL4B column (Pharmacia Inc).

BAL sediment was suspended in TRIS buffer and after vigorous vortexing drops of the suspension were placed on premarked areas on 


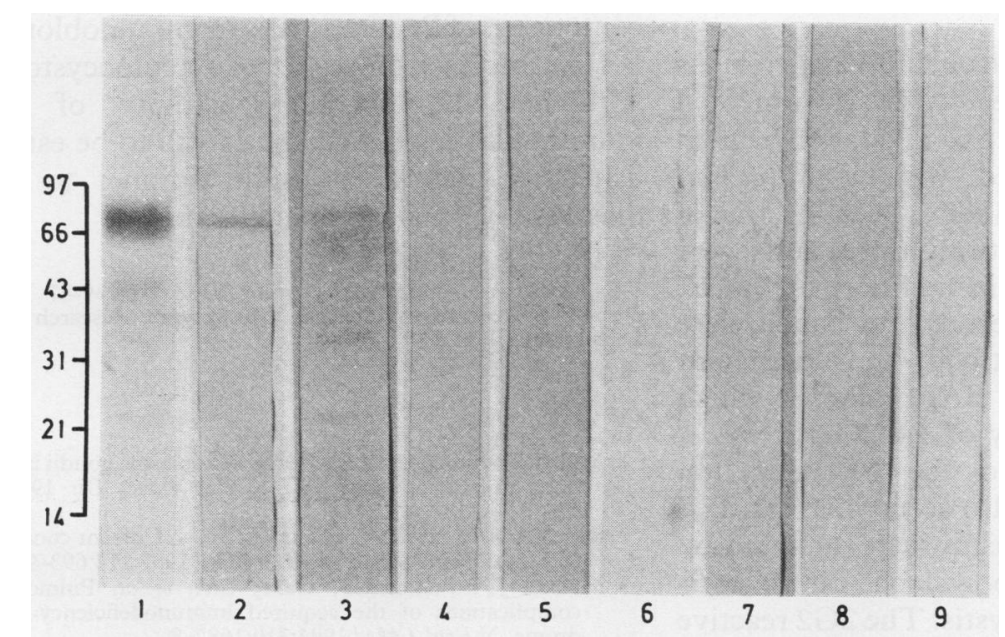

Figure 1 Representative blots of $B A L$ supernatants from three individual patients with $P$ carinii, reacted with monoclonal antibody 2G2, specific for human Pneumocystis (strips 1,2, and 3). Note single distinct band in lanes 1 and 2, and three bands in lane 3. Strips 4, 5, and 6 were reacted with monoclonal antibody 2C11, specific only for Pneumocytis of rat origin. Strips 7, 8, and 9 represent conjugate controls. Numbers on left indicate molecular weights of protein standards (in thousands).

Figure 2 Blots of purified human Pneumocystis organisms developed with rabbit antiPneumocystis polyclonal antibodies (strip 2), with normal rabbit serum (strip 1). Numbers on left indicate the molecular weights of protein standards (in thousands).

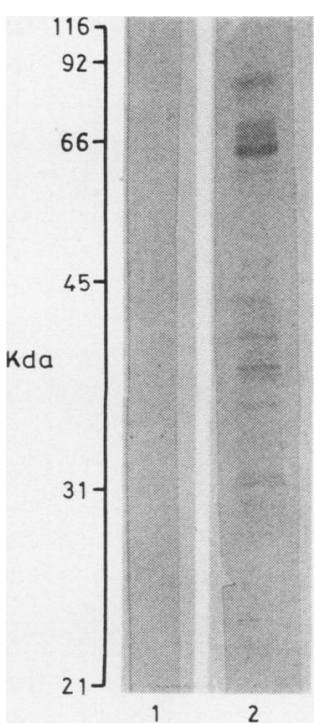

glass slides. The drops were spread, air dried, and processed for staining by a modification of the Grocott's silver methenamine stain. For immunofluorescence studies, a commercially available anti- $P$ carinii monoclonal antibody reagent (Progen Biotechnic, Heidelberg) was used and the staining performed according to the manufacturer's instructions.

$P$ carinii organisms purified from the BAL sample of a patient with AIDS were sonicated in the presence of $0.125 \mathrm{M}$ TRIS- $\mathrm{HCl}$ buffer and suspended in $2 \%$ sodium dodecyl sulphate (SDS) sample buffer ${ }^{14}$ containing $50 \mathrm{mM}$ dithiothreiotol, $10 \mathrm{mM}$ ' EDTA, $1 \mathrm{mM}$ leupeptin, $1 \mathrm{mM}$ phenylmethylsulfonyl and $0.01 \%(\mathrm{w} / \mathrm{v})$ bromophenol-blue fluoride to a final concentration of $1 \mathrm{mg}$ protein $/ \mathrm{ml}$. After boiling, the sample was centrifuged and the supernatant either stored $\left(-70^{\circ} \mathrm{C}\right)$ or applied to $5-15 \%$ discontinuous SDS-polyacrylamide gels-SDS-PAGE-(10 $\mu$ g protein/well). BAL samples were diluted $1 / 2$ with $2 \%$ SDS sample buffer, boiled, and $25 \mu \mathrm{l}$ applied to each lane. After electrophoresis the separated proteins were transferred to nitrocellulose paper electrophoretically using Bio-Rad Trans-Blot apparatus. On completion of the transfer, the nitrocellulose sheets were blocked by incubation for 30 minutes at $37^{\circ} \mathrm{C}$ with $5 \%$ non-fat dry milk in phosphate buffered saline (PBS). The sheets were washed with TRIS-buffered saline containing $0.05 \%$ Tween 20 . The strips were incubated for two hours in the presence of $2 \mathrm{ml}$ of $1 / 100$ dilution of purified anti- $P$ carinii monoclonal antibody $2 \mathrm{G} 2$ or $1 / 50$ dilution of a rabbit anti-P carinii IgG. The antibodies were diluted in 5\% non-fat dry milk in PBS containing $0.05 \%$ Tween 20 . After washing, the nitrocellulose strips were incubated $\left(37^{\circ} \mathrm{C}\right.$ for 30 minutes) with a diluted preparation of detecting antibodies-that is, horseradish peroxidase conjugated goat antibody to mouse IgG or rabbit IgG. After rewashing, the strips were incubated with substrate 4-chloro-1nephthol $(0.05 \%)$ plus $0.015 \% \mathrm{H}_{2} \mathrm{O}_{2}$. Controls included testing of direct binding of conjugates to antigen or BAL samples.

\section{Results}

Sediments prepared from BAL specimens of 26 patients with AIDS and clinical manifestations of $P$ carinii pneumonia contained intact organisms shown by methenamine silver stain or a monoclonal antibody based immunofluorescence antibody test. Remarkably high numbérs of cysts ( 10 clusters of cysts a smear) were identified in the BAL sediments of 20 of the 26 cases by both staining procedures. In the six remaining samples fewer than five small clusters of cysts a smear were detectable with the immunofluorescence antibody but none by silver stain. Four of these six patients were receiving aerosol pentamidine treatment when the BAL sample was collected. The other two patients had been receiving antifungal treatment, but developed neutropenia, and treatment was discontinued. In four of these six cases sediments of BAL samples collected initially were negative but sediments from second specimens obtained from the same four patients seven to 10 days later proved positive.

Supernatants of BAL samples from each of the 26 cases with documented pneumocystosis and from 10 controls were examined for the presence of soluble $P$ carinii antigen(s) by immunoblotting using an anti- $P$ carinii monoclonal antibody, $2 \mathrm{G} 2$, as the detection probe. Immunoblots of the supernatants from all the 26 samples from patients with $P$ carinii pneumonia showed a distinct band in the region of the 67 kilodalton molecular weight marker (fig 1). Considerable variation was observed in the quantity of $2 \mathrm{G} 2$ antibody binding to the antigenic component in individual BAL supernatants, indicated by the intensity of 67 kilodaltons bands which varied from intense (14 samples) to faint (12 samples). Interestingly, in the supernatant blots of four BAL samples $2 \mathrm{G} 2$ antibody identified multiple bands besides the 67 kilodalton component. No specific reactivity was visible when identical blots were reacted with another monoclonal antibody-namely, 2C11 which is specific for $P$ carinii of rat origin. Furthermore, no specific bands were identified by $2 \mathrm{G} 2$ antibody in blots of supernatants from 10 control BAL $M$ specimens.

SDS-PAGE of purified $P$ carinii organisms of human origin resulted in the separation of the individual polypeptides of the organisms. Blots developed with rabbit anti-P carinii polyclonal antibodies showed distinct antigens of Pneumocystis including a band of about 67 kilodaltons. Apparently, the 67 kilodalton band identified by polyclonal antiserum in blots prepared from purified organisms corresponds to that observed on BAL supernatant blots probed with $2 \mathrm{G} 2$ antibody. No bands appeared on blots of purified Pneumocystis organisms reacted with control rabbit IgG (fig 2). 


\section{Discussion}

Supernatants from a total of $26 \mathrm{BAL}$ specimens originating from cases with documented pneumocystosis showed a 67 kilodalton band in immunoblots developed with a $P$ carinii specific monoclonal antibody, 2G2. In four of the $26 \mathrm{BAL}$ immunoblots multiple bands were consistently observed, in addition to the 67 kilodalton band. Conversely, no bands were recognised by $2 \mathrm{G} 2$ antibody in supernatant blots from 10 control BAL samples which illustrates the specificity of the $2 \mathrm{G} 2$ prove. A band corresponding to the 67 kilodalton molecular weight was also seen when purified human $P$ carinnii organsms were analysed by immunoblotting with polyclonal rabbit antiserum against Pneumocystis. The $2 \mathrm{G} 2$ reactive 67 kilodalton antigenic component identified in supernatants of BAL samples is probably identical with the antigen recognised in protein blots of purified organisms by $P$ carinii specific polyclonal antibodies in rabbits. The detection of 2 G2 antibody reactive soluble Pneumocystis antigen(s) in supernatants of unconcentrated BAL specimens suggests that this antigenic component is released in appreciable quantities in patients with acute disease. Although there is a potential for carryover of intact organisms when supernatants are harvested from BAL samples, this was not observed after rigorous examination by a variety of staining procedures. There were four separate BAL specimens from which the sediments proved negative for Pneumocystis organisms on initial examination, and $2 \mathrm{G} 2$ reactive soluble antigen could be identified in the same samples. Whether other soluble antigenic molecules of $P$ carinii also exist in BAL samples of patients with pneumocystosis is not known and requires investigation. In conclusion, $2 \mathrm{G} 2$ monoclonal antibody specific for $P$ carinii of human origin facilitated the detection of a soluble antigenic component of Pneumocystis in immunoblots of BAL samples from cases of pneumocystosis. Although the overall sensitivity of the immunoblotting procedure is still to be established, it may be a useful adjunct to the diagnosis of $P$ carinii pneumonia. This work was supported by grant FKZ: II-039-87 from
the AIDS Program of German Ministry of Research and Technology (BMFT)

1 Mills J. Pneumocystis carinii and Toxoplasma gondii infections in patients with AIDS, Rev Infect Dis 1986;8: 1001-11.

2 Salfelder K, Schwarz J. Pneumocystosis. Current concepts and recent advances. Am J Dis Child 1967;114:693-9.

3 Murray JF, Felton CP, Garay SM, et al. Pulmonary complications of the acquired immunodeficiency syncomplications of the acquired immus.
drome. N Engl J Med 1984;310:1682-8.

4 Cushion MT, Walzer PD. Growth and serial passage of Pneumocystis carinii in A 549 cell line. Infect Immu 1984;44:245-51.

5 Bartlett MS, Verbanc PA, Smith JW. Cultivation of Pneumocystis carinii within WI-38 cells. J Clin Microbiol 1979;10:796-9.

6 Pifer L, Hughes WT, Murphy MJ. Propagation of Pneumocystis carinii in vitro. Pediatr Res 1977;11:305-16.

7 Walzer PD. Diagnosis of Pneumocystis carinii pneumonia. $J$ Infect Dis 1988;157:629-32.

8 Pesanti EL. Pneumocystis carinii. Clin Microbiol Lett 1987;9:133-8.

9 Kovacs JA, Ng VL, Masur $\mathrm{H}$, et al. Diagnosis of Pneumocystis carinii pneumonia: Improved detection in sputum with use of monoclonal antibodies. $N$ Engl $J$ Med 1988;318:589-93.

10 Meyers JD, Pifer LL, Sales GE, Thomas ED. The value of Pneumocystis carinii antibody and antigen detection for diagnosis of Pneumocystis carinii pneumonia after marrow transplantation. Am Rev Respir Dis 1978;120:1283-7.

11 Pifer LW, Wolf BL, Weems JJ, Woods DR, Edwards CC Joyner RE. Pneumocystis carinii anti genemia in acquired Joyner RE. Priency 1988;26:1357-61.

12 Tanabe $K$, Furuta $T$. Detection of circulating antigens of Pneumocystis carinii in human sera by a sandwich
enzyme-immunoassay. Zentral Bakteriol Parasitenkd enzyme-immunoassay. Zentral Bakteri

13 Maddison SE, Walls KW, Haverkos HW, Juranek DD Evaluation of serologic tests for Pneumocystis carini antibody and antigenemia in patients with acquired immunodeficiency syndrome. Diagn Microbiol Infect Dis 1984;2:69-73.

14 Laemmli UK. Cleavage of structural proteins during the assembly of the head of bacteriophage T 4. Nature (Lond) 1970;227:680-5. 